\title{
Symmetries in two-dimensional dilaton gravity with matter
}

\author{
Miguel Navarro* \\ Instituto de Matemáticas y Física Fundamental, CSIC, Serrano 113-123, 28006 Madrid, Spain \\ and Instituto Carlos I de Física Teórica y Computacional, Facultad de Ciencias, Universidad de Granada, \\ Campus de Fuentenueva, 18002, Granada, Spain
}

(Received 30 June 1997)

\begin{abstract}
The symmetries of generic two-dimensional (2D) dilaton models of gravity with (and without) matter are studied in some detail. It is shown that $\delta_{2}$, one of the symmetries of the matterless models, can be generalized to the case where matter fields of any kind are present. The general (classical) solution for some of these models, in particular, those coupled to chiral matter, which generalizes the Vaidya solution of Einstein Gravity, is also given. [S0556-2821(97)00124-0]

PACS number(s): 04.60.Kz, 04.50. $+\mathrm{h}, 11.30 .-\mathrm{j}$
\end{abstract}

\section{INTRODUCTION}

At present, one of the main challenges of theoretical physics is to devise a quantum theory which will provide a complete description of gravity. Because of the complexity of the theories involved, the Einstein-Hilbert gravity theory, for instance, this task faces imposing technical difficulties. However, equally important or even more serious are the conceptual problems that arise. These are not only due to a variety of unfamiliar features which are peculiar to diffeomorphisminvariant theories but are also due to our present lack of an adequate formulation and interpretation of quantum mechanics. To a large extent, we ignore what a quantum theory really is-i.e., we ignore what the adjective "quantum" really means - and how we should interpret these theories. Because of that, we ignore not only how to produce a quantum theory of gravity but also what would constitute a successful completion of this task.

In this context "toy theories" should have a crucial concept-clarifying role to play; no wonder a variety of them are currently under study. Prominent among them are the two-dimensional (2D) dilaton models of gravity, which are two-dimensional general-covariant models whose gravity sector involves, along with the space-time metric $g_{\mu \nu}$, a scalar field $\phi$, the dilaton (for a review, see Ref. [1]). These models, while much simpler to handle than are their higherdimensional cousins, share with them not only the conceptual problems which are peculiar to diffeomorphisminvariant theories but also their most relevant physical features, such as the formation of black holes and their subsequent evaporation. Some of these models can be obtained, via dimensional reduction, from realist, higher-dimensional theories, but this is not a necessary, nor even convenient, fact to bear in mind when approaching these models, as they may be regarded as something similar to two-dimensional BransDicke theories.

If attention is restricted to the usual category of modelsthat is, those with second-order Euler-Lagrange equations of motion-a very useful result to take into account is that,

\footnotetext{
*Electronic address: mnavarro@ugr.es; URL:http://www.ugr.es/ $\sim$ mnavarro
}

excepted isolated pathologies which may arise, the actions of these models can all be brought, by means of appropriate redefinitions of the dilaton field and conformal redefinitions of the metric, to the generic form $[3,4]$

$$
S_{\mathrm{GDG}}=S_{V}-S_{M},
$$

where

$$
S_{V}=\int d^{2} x \sqrt{-g}[R \phi+V(\phi)]
$$

and $S_{M}$ is a gravity-matter interaction term which may involve the dilaton field as well as the metric.

Despite their being much simpler that their higherdimensional cousins, few of these models have been solved classically, let alone quantum mechanically, when matter fields are present. As is well known, solvability, classical or quantum, is usually related to the presence of invariances, which is the reason that classical solvability usually implies quantum solvability. Notwithstanding this fact, little attention had been paid to the symmetries of these models, apart from the conformal ones (see, however, Ref. [2]). Recently, a large variety of symmetries, which are in general nonconformal, have been uncovered for the matterless 2D dilaton models and they have been shown to explain the (classical) solvability of these theories [5].

In the present paper, we shall consider the generic 2D dilaton gravity with matter, Eq. (1), and shall explore how much of what has been done in the matterless case can be extended to theories with matter. Nonetheless, to make the present paper as self-contained as possible and to make it clear how the symmetries actually underlie the solvability of these models, a brief but systematic view of the matterless theories shall be presented in Sec. II. It is worth discussing beforehand some general features of these models.

The general variation of the Lagrangian in Eq. (1) yields

$$
\begin{aligned}
\delta \mathcal{L}= & \sqrt{-g}\left\{\left[R+V^{\prime}(\phi)-T_{\phi}\right] \delta \phi+\left[g_{\mu \nu} \square \phi-\frac{1}{2} g_{\mu \nu} V(\phi)\right.\right. \\
& \left.-\nabla_{\mu} \nabla_{\nu} \phi-T_{\mu \nu}\right] \delta g^{\mu \nu}+(E-L)_{A} \delta f^{A}-\nabla_{\alpha} s^{\alpha},
\end{aligned}
$$


where $\nabla_{\alpha} s^{\alpha}$ includes all the terms that appear due to the "integrations by part", which are required to produce the equations of motion.

The equations of motion can be brought to the form

$$
\begin{gathered}
R+V^{\prime}(\phi)=T_{\phi}, \\
\square \phi=V+T, \\
\nabla_{\mu} \nabla_{\nu} \phi=\frac{1}{2} g_{\mu \nu} V+g_{\mu \nu} T-T_{\mu \nu}, \\
(E-L)_{A}=0,
\end{gathered}
$$

where $T$ is the trace of $T_{\mu \nu}$.

Although much of our present study is meant to apply to all kinds of matter, we shall exemplify much of our developments with a massless scalar field $\xi$, with action

$$
S_{M}=\frac{1}{2} \int d^{2} x \sqrt{-g} \Omega(\phi)(\nabla \xi)^{2}
$$

and unspecified function $\Omega$. (The particular case of dimensionally reduced spherically symmetric Einstein-Hilbert gravity minimally coupled to a massless scalar field is recovered with $V=2 / \sqrt{\phi}$ and $\Omega=G \phi$, with $G$ the Newton constant.)

For this example, we have

$$
\begin{gathered}
T_{\phi}=\frac{\Omega^{\prime}(\phi)}{2}(\nabla \xi)^{2}, \\
T_{\mu \nu}=\frac{\Omega}{2}\left\{\nabla_{\mu} \xi \nabla_{\nu} \xi-\frac{1}{2} g_{\mu \nu}(\nabla \xi)^{2}\right\} .
\end{gathered}
$$

Let us now go back to Eq. (4). It is apparent that the second equation is redundant as it follows from the third one. However, the same is true for the first one. To show this let us first indicate that invariance under diffeomorphisms of $S_{M}$ implies that, when the equations of motion of the matter fields are satisfied, the following generalized conservation law for the energy-momentum tensor $T_{\mu \nu}$ must be satisfied:

$$
2 \nabla^{\nu} T_{\mu \nu}+T_{\phi} \nabla_{\mu} \phi=0,
$$

which implies

$$
T_{\phi}=-\frac{2}{(\nabla \phi)^{2}} \nabla^{\mu} \phi \nabla^{\nu} T_{\mu \nu}
$$

On the other hand, the affine connection $\nabla_{\mu}$ and the Riemann tensor $R_{\alpha \mu \beta \nu}$ obey

$$
\left[\nabla_{\nu}, \nabla_{\beta}\right] \zeta_{\mu}=-\zeta_{\rho} R_{\mu \beta \nu}^{\rho}
$$

for any vector field $\zeta_{\mu}$. Particularizing this equality to $\zeta_{\mu}$ $=\nabla_{\mu} \phi$ and using the fact that in two-dimensions $R_{\mu \nu}$ $=\frac{1}{2} g_{\mu \nu} R$ yield, after a bit of algebra,

$$
R \nabla_{\mu} \phi+V^{\prime} \nabla_{\mu} \phi=-2 \nabla^{\nu} T_{\mu \nu},
$$

which, together with Eq. (8), yields the desired equality.
Therefore, all the equations of motion of the theory are encapsulated in the last and next-to-last equations in Eq. (4). As we shall see, this result facilitates finding the general classical trajectories.

Given that in the models we are dealing with it is easier to find the conserved currents than the associated (Noether) symmetries, let us remind the reader, before proceeding, of the Noether theorem, suitably taylored for the occasion. Let $\mathcal{L}=\mathcal{L}\left(\Psi^{a}\right)$ be an arbitrary Lagrangian with general variation $\delta \mathcal{L}=(E-L)_{a} \delta \Psi^{a}-\nabla_{\mu} s^{\mu}$. Let $j_{0}^{\mu}$ be a current which is conserved on shell and $\delta_{0} \Psi^{a}$ a transformation of the fields. Then $j_{0}^{\mu}$ is the Noether current associated to $\delta_{0} \Psi^{a}$ iff, without using the equations of motion, the following equality holds as an identity:

$$
(E-L)_{a} \delta_{0} \Psi^{a}=\nabla_{\mu} j_{0}^{\mu} .
$$

In general, due to semi-invariance, the current $j_{0}^{\mu}$ will not equal $s^{\mu}\left(\delta_{0} \Psi^{a}\right)$.

\section{SYMMETRIES AND GENERAL SOLUTION FOR THE MATTER-LESS THEORIES}

With the scalars $\phi$ and $\varphi \equiv(\nabla \phi)^{2}$ and the vector field $\nabla_{\mu} \phi$, we can build scalar vector and tensor fields and we can check whether or not they are conserved. Let $J(\phi)$ be a primitive of $V, d J / d \phi=V$. It can be shown that the following results hold.

Conserved scalars. The local energy $[2,4]$

$$
E=\frac{1}{2}\left[(\nabla \phi)^{2}-J\right]
$$

is a conserved scalar: $\nabla_{\mu} E=0$, and consequently $f(E)$ is also for any function $f$. Moreover, these are the only conserved scalars which can be constructed with $\phi$ and $(\nabla \phi)^{2}$.

The Noether symmetry associated to $E$ is given by

$$
\delta_{a} \phi=0, \quad \delta_{a} g_{\mu \nu}=g_{\mu \nu} a_{\sigma} \nabla^{\sigma} \phi-\frac{1}{2}\left(a_{\mu} \nabla_{\nu} \phi+a_{\nu} \nabla_{\mu} \phi\right)
$$

with arbitrary constant bivector $a^{\mu}$.

Conserved currents. The conserved currents of the form $j^{\mu}=A(\phi, \varphi) \nabla^{\mu} \phi$ can all be written as

$$
j_{f}^{\mu}=f(E) \frac{\nabla^{\mu} \phi}{(\nabla \phi)^{2}}
$$

for some function $f$. The associated symmetries are

$$
\begin{gathered}
\delta_{f} \phi=0 \\
\delta_{f} g_{\mu \nu}=-\epsilon f^{\prime}(E)\left(g_{\mu \nu}-\frac{\nabla_{\mu} \phi \nabla_{\nu} \phi}{(\nabla \phi)^{2}}\right)+\epsilon f(E)\left(\frac{g_{\mu \nu}}{(\nabla \phi)^{2}}\right. \\
\left.-2 \frac{\nabla_{\mu} \phi \nabla_{\nu} \phi}{(\nabla \phi)^{4}}\right) .
\end{gathered}
$$

In particular, for $f=1$, the corresponding current and symmetry are, respectively, 


$$
\begin{gathered}
j_{1}^{\mu}=\frac{\nabla^{\mu} \phi}{(\nabla \phi)^{2}}, \\
\delta_{1} \phi=0, \quad \delta_{1} g_{\mu \nu}=\epsilon\left(\frac{g_{\mu \nu}}{(\nabla \phi)^{2}}-2 \frac{\nabla_{\mu} \phi \nabla_{\nu} \phi}{(\nabla \phi)^{4}}\right) .
\end{gathered}
$$

Now, let $j_{R}^{\mu}$ be defined by $\nabla_{\mu} j_{R}^{\mu}=R$. It is easy to see that the following current is conserved:

$$
j_{2}^{\mu}=j_{R}^{\mu}+V \frac{\nabla^{\mu} \phi}{(\nabla \phi)^{2}}
$$

with symmetry

$$
\delta_{2} \phi=\epsilon, \quad \delta_{2} g_{\mu \nu}=\epsilon V\left(\frac{g_{\mu \nu}}{(\nabla \phi)^{2}}-2 \frac{\nabla_{\mu} \phi \nabla_{\nu} \phi}{(\nabla \phi)^{4}}\right) .
$$

Conserved tensors. There exists a great variety of conserved two-tensors of the form

$$
S^{\mu \nu}=A(\phi, \varphi) \nabla^{\mu} \phi \nabla^{\nu} \phi+B(\phi, \varphi) g^{\mu \nu} .
$$

They are given by the general solution of the equation $\left(A_{J}\right.$ $\left.=d A / d J, A_{\varphi}=d A / d \varphi\right)$

$$
\frac{3}{2} A+A_{J} \varphi+A_{\varphi} \phi+B_{J}+B_{J}=0
$$

which implies that $A(B)$ can be written as $A=A(J, \varphi)[B$ $=B(J, \varphi)]$. For $A=0$, we recover the conserved scalars described above. Another useful solution is the following traceless tensor, which is basically unique:

$$
S^{\mu \nu}=\frac{\nabla^{\mu} \phi \nabla^{\nu} \phi}{(\nabla \phi)^{4}}-\frac{1}{2} \frac{g^{\mu \nu}}{(\nabla \phi)^{2}} .
$$

General local solution. Once the symmetries are at our disposal, it is easy to solve the equations of motion. It can be shown [5] that the following "metric", is invariant under $\delta_{2}$ :

$$
\bar{g}_{\mu \nu}=\frac{1}{(\nabla \phi)^{2}}\left(g_{\mu \nu}-\frac{\nabla_{\mu} \phi \nabla_{\nu} \phi}{(\nabla \phi)^{2}}\right) \text {, }
$$

which implies

$$
g_{\mu \nu}=(\nabla \phi)^{2} \bar{g}_{\mu \nu}+\frac{\nabla_{\mu} \phi \nabla_{\nu} \phi}{(\nabla \phi)^{2}}
$$

As $\nabla_{\mu} \phi \bar{g}_{\mu \nu}=0$, we must have

$$
\bar{g}_{\mu \nu}=A k_{\mu} k_{\nu}
$$

with $k^{\mu}$ the vector density:

$$
k^{\mu}=\frac{\epsilon^{\mu \nu}}{\sqrt{-g}} \nabla_{\nu} \phi
$$

As $\nabla_{\mu} k_{\nu}=\nabla_{\nu} k_{\mu}, k_{\mu}$ is, at least locally, a total derivative: $k_{\mu}=\nabla_{\mu} t$ for some function $t$, it is then natural to choose as local coordinates $r \equiv \phi$ and $t$. Now, it is easy to see that $k^{\mu}$ is a Killing vector [4], which implies $A=A(r)$. The equations of motion (4) imply $A=-1$. Therefore, we finally arrive at the general solution

$$
\begin{gathered}
\phi=r, \\
g_{\mu \nu}=[2 M-J(r)] \nabla_{\mu} t \nabla_{\nu} t-\frac{\nabla_{\mu} r \nabla_{\nu} r}{2 M-J(r)},
\end{gathered}
$$

where $M=-E$ is an arbitrary constant.

Another elegant way of arriving at the general solutions is by using free-field methods. Here we choose the conformal gauge

$$
d s^{2}=2 e^{\rho} d x^{+} d x^{-}
$$

for which we have $R=-2 e^{-\rho} \partial_{+} \partial_{-} \rho$ and $\square=2 e^{-\rho} \partial_{+} \partial_{-}$.

In the conformal gauge, the conservation law for the traceless tensor in Eq. (20) takes the form

$0=\partial_{+} T_{--}=\partial_{+}\left(\frac{\partial_{-} \phi \partial_{-} \phi}{(\nabla \phi)^{4}}\right), \quad 0=\partial_{-} T_{++}=\partial_{-}\left(\frac{\partial_{+} \phi \partial_{+} \phi}{(\nabla \phi)^{4}}\right)$

which imply $\left(\partial_{+} \phi\right) /(\nabla \phi)^{2}=\partial_{+} p, \quad\left(\partial_{-} \phi\right) /(\nabla \phi)^{2}=\partial_{-} m$, for some $p=p\left(x^{+}\right)$, and $m=m\left(x^{-}\right)$.

A bit of algebra leads us to

$$
e^{\rho}=2(\nabla \phi)^{2} \partial_{+} p \partial_{-} m=2(2 E+J) \partial_{+} p \partial_{-} m .
$$

By fixing the residual gauge as $p=\frac{1}{2} x^{+}, m=-\frac{1}{2} x^{-}$, we finally arrive at

$$
\begin{gathered}
\int^{\phi} \frac{d \tau}{2 M-J(\tau)}=-\frac{1}{2}\left(x^{+}-x^{-}\right), \\
e^{\rho}=\frac{1}{2}(2 M-J) .
\end{gathered}
$$

\section{SYMMETRIES IN 2D DILATON GRAVITY WITH MATTER}

Let us now introduce matter fields. Our most general result in this case is that the symmetry (current) $\delta_{2}\left(j_{2}^{\mu}\right)$ above can be generalized for whatever kind of matter is present. More precisely, if $S_{M}$ is invariant under diffeomorphisms and the equations of motion (4) are obeyed, the following current is conserved:

$$
j_{2}^{\mu}=j_{R}^{\mu}+V \frac{\nabla^{\mu} \phi}{(\nabla \phi)^{2}}+2 \frac{\nabla_{\nu} \phi}{(\nabla \phi)^{2}} T^{\mu \nu} .
$$

For us to show this, it suffices to make use of the equations of motion (4), Eq. (8), and of the fact that in two dimensions, and for any tensor $N_{\mu}^{\nu}$, the following quantity vanishes identically $\left(N=N_{\rho}^{\rho}\right)$ :

$$
\begin{aligned}
& \left(N_{\alpha}^{\mu}-\frac{1}{2} \delta^{\mu}{ }_{\alpha} N\right)\left(N^{\alpha}{ }_{\nu}-\frac{1}{2} \delta^{\alpha}{ }_{\nu} N\right) \\
& \quad-\frac{1}{2} \delta^{\nu}{ }_{\mu}\left(N^{\beta}{ }_{\alpha}-\frac{1}{2} \delta^{\beta}{ }_{\alpha} N\right)\left(N^{\alpha}{ }_{\beta}-\frac{1}{2} \delta^{\alpha}{ }_{\beta} N\right)
\end{aligned}
$$

or, equivalently, 


$$
N_{\mu}^{\alpha} N_{\alpha}^{\nu}=\frac{1}{2} \delta_{\mu}^{\nu} N_{\alpha}^{\beta} N_{\beta}^{\alpha}+N N_{\mu}^{\nu}-\frac{1}{2} \delta_{\mu}^{\nu} N^{2}
$$

The associated Noether symmetry is

$$
\begin{gathered}
\delta_{2} \phi=\epsilon \\
\delta_{2} g_{\mu \nu}=\epsilon\left\{V\left(\frac{g_{\mu \nu}}{(\nabla \phi)^{2}}-2 \frac{\nabla_{\mu} \phi \nabla_{\nu} \phi}{(\nabla \phi)^{4}}\right)+2 \frac{T_{\mu \nu}}{(\nabla \phi)^{2}}-2 T \frac{g_{\mu \nu}}{(\nabla \phi)^{2}}\right. \\
-\frac{2}{(\nabla \phi)^{4}}\left(\nabla_{\nu} \phi \nabla^{\alpha} \phi T_{\alpha \mu}+\nabla_{\mu} \phi \nabla^{\alpha} \phi T_{\alpha \nu}\right) \\
\left.+4 \frac{g_{\mu \nu}}{(\nabla \phi)^{4}} \nabla^{\alpha} \phi \nabla^{\beta} \phi T_{\alpha \beta}\right\} .
\end{gathered}
$$

The variation $\delta f^{A}$ of the matter fields is such that, off shell,

$$
(E-L)_{A} \delta f^{A}=2 \frac{\nabla_{\nu} \phi}{(\nabla \phi)^{2}} \nabla_{\mu} T^{\mu \nu} .
$$

For our exemplifying scalar matter field, this transformation is

$$
\delta \xi=\epsilon \frac{\nabla^{\mu} \phi}{(\nabla \phi)^{2}} \nabla_{\mu} \xi=\epsilon \frac{\nabla \phi \nabla \xi}{(\nabla \phi)^{2}} .
$$

It is easy to see that, in spite of what happens in the matterless case, this symmetry does not correspond to the diffeomorphism generated on-shell by the vector field $s^{\mu}$ $=\nabla^{\mu} \phi /(\nabla \phi)^{2}$.

Let us now consider currents of the form

$$
S^{\mu}=A(\phi, \varphi) \nabla^{\mu} \phi+B(\phi, \varphi) \nabla_{\nu} T^{\mu \nu} .
$$

Conservation implies

$$
\begin{aligned}
0= & A_{\phi}(\nabla \phi)^{2}+A_{\varphi} V(\nabla \phi)^{2}+A V-\frac{1}{2} B(\nabla \phi)^{2} T_{\phi} \\
& +\left[2 A_{\varphi}(\nabla \phi)^{2}+A+\frac{1}{2} B V\right] T+\left[B+B_{\varphi}(\nabla \phi)^{2}\right]\left(T^{2}\right. \\
& \left.-T_{\alpha \beta} T^{\alpha \beta}\right)+\left[-2 A_{\varphi}+B_{\phi}+B_{\varphi} V\right] \nabla_{\mu} \phi \nabla_{\nu} \phi T^{\mu \nu},
\end{aligned}
$$

where Eq. (26) has been used. The following can be shown to be true. (1) If $T_{\phi}=0, T=0$ and $V^{\prime}=\beta V$ (with $\beta=$ const) the following current is conserved:

$$
S_{\beta}^{\mu}=-\beta \nabla^{\mu} \phi+V \frac{\nabla^{\mu} \phi}{(\nabla \phi)^{2}}+2 \frac{\nabla_{\nu} \phi}{(\nabla \phi)^{2}} T^{\mu \nu} .
$$

The Noether symmetry associated to $j_{\beta}^{\mu}=j_{2}^{\mu}-S_{\beta}^{\mu}$ is conformal and is given by [5]

$$
\delta_{\beta} \phi=\epsilon, \quad \delta g_{\mu \nu}=-\epsilon \beta g_{\mu \nu}, \quad \delta f^{A}=0 .
$$

(2) If $T_{\phi}=0, T=0$, and $T_{\alpha \beta} T^{\alpha \beta}=0$ (chiral matter),

$$
S_{f}^{\mu}=f(E) \nabla_{\nu} \phi T^{\mu \nu}
$$

is a conserved current for any function $f$. The associated symmetry transformation of the gravity sector is

$$
\delta \phi=0, \quad \delta g_{\mu \nu}=\varepsilon T_{\mu \nu} .
$$

Conserved two-tensors. Consider now (symmetric) tensor fields of the form

$$
S^{\mu \nu}=A(\phi, \varphi) \nabla^{\mu} \phi \nabla^{\nu} \phi+B(\phi, \varphi) g^{\mu \nu}+C(\phi, \varphi) T^{\mu \nu} .
$$

It can be shown that for $T_{\phi}=0$ and $T=0$ the tensor fields with

$$
\begin{gathered}
C=C(\phi), \quad C \text { an arbitrary function, } \\
B=\left(C_{\phi}+\alpha\right) \varphi+2 \alpha J+\frac{1}{2} \int^{\phi} V C_{\phi}, \\
A=-C_{\phi}-2 \alpha
\end{gathered}
$$

are conserved. If $T \neq 0$, these tensors are still conserved if $\alpha=0$. The tensor with $C=\phi, \alpha=-\frac{1}{2}$,

$$
J^{\mu \nu}=\frac{1}{2} g^{\mu \nu}\left[(\nabla \phi)^{2}-J(\phi)\right]+\phi T^{\mu \nu}
$$

can be regarded as the generalization of the local energy of the massless models. The conserved tensor with $C=0$ is purely kinematical-it does not depend on the matter fields:

$$
S_{0}^{\mu \nu}=\nabla^{\mu} \phi \nabla^{\nu} \phi-\frac{1}{2} g^{\mu \nu}(\nabla \phi)^{2}-g^{\mu \nu} J
$$

Chiral matter. We define chiral matter as the one whose energy-momentum tensor obeys

$$
T_{\phi}=0, \quad T_{\mu}^{\mu}=0, \quad T_{\mu \nu} T^{\mu \nu}=0 .
$$

In the conformal gauge we have

$$
0=T_{\mu \nu} T^{\mu \nu}=2 e^{-2 \rho} T_{++} T_{--} .
$$

Therefore the set of all solution splits in two sectors of leftmoving and right-moving fields. Let us choose $T_{--}=0$. The conservation law for the tensor in Eq. (39) implies

$$
\begin{gathered}
\partial_{-} E=0 \Rightarrow E=P\left(x^{+}\right), \\
\partial_{+} E+\nabla^{+} \phi T_{++}=0 \Rightarrow \nabla^{+} \phi=p\left(x^{+}\right) .
\end{gathered}
$$

Thus, we are led to the equations

$$
\begin{gathered}
e^{-\rho} \partial_{-} \phi=p\left(x^{+}\right), \\
\partial_{+} \phi=\frac{P}{p}+\frac{1}{2 p} J, \\
\frac{\partial_{+} P}{p}=-T_{++} .
\end{gathered}
$$

However, it does not appear that, in the present coordinates, these equations can be solved in general for arbitrary $J$ and $T_{++}$. To go further, let us make a change of coordinates

$$
x^{+}=u, \quad x^{-}=x^{-}(u, r) .
$$


so that $\phi \equiv r$. The metric and the energy-momentum tensor in this coordinates takes the form

$$
\begin{gathered}
d s^{2}=e^{\lambda}\left[2 d \phi d u+e^{\lambda} A(d u)^{2}\right], \\
T=T_{u u}(d u)^{2}
\end{gathered}
$$

with $T_{u u}=T_{u u}(u)$. Now, by writing down in this gauge the equations of motion (4), it is easy to show that $e^{\lambda}$ is pure gauge, and finally arrive at the following general solution:

$$
d s^{2}=2 d r d u+[2 M(u)-J](d u)^{2},
$$

with

$$
M(u)=\int^{u} \mathrm{~d} \tilde{u} T_{u u}(\tilde{u}) .
$$

This metric generalizes, for arbitrary potential $V$, the Vaidya solution of Einstein gravity.

\section{CONCLUSIONS}

We have analyzed in a rather systematic way the conserved currents and the symmetries of the 2D dilaton models of gravity with and without matter. In particular we have shown that $\delta_{2}$ can be extended to models coupled to any kind of matter. We have also shown how analytic solvability is directly related with the existence of invariances-and hence of conserved currents. In fact, almost all the models which are known to be solvable fall into one of the categories of symmetric models that we have described in the present paper. The Jackiw-Teitelboim model, $V=4 \lambda^{2} \phi$, coupled to conformal matter may be regarded as an exception to this rule, as it is solvable [6] even though, apart from $\delta_{2}$, it is not known to have any additional symmetry. This model is solvable because, in the conformal gauge, the first equation in Eq. (4) is a Liouville equation. This Liouville equation, despite being a limiting case of the free-field equation of the exponential model which is associated to the symmetry in Eq. (34) (see also Ref. [5]), does not appear to be related itself to any invariance. In fact, the whole problem of solving the generic 2D dilaton models of gravity (with conformal matter) can be regarded as a generalization of Liouville theory. It would be interesting to see if the machinery of integrable systems is useful here.

The massless 2D dilaton models have been shown to be related to Poisson- $\sigma$ models, and this seems to explains their highly symmetric nature (see, for instance, Refs. [7,8] and references therein). On the other hand, the underlying reason why $\delta_{2}$ is so general remains a mystery to us as of the writing of this paper. It seems to indicate that these models have a degree of unity previously unexpected. This unity may have important consequences in relation to their solvability.

\section{ACKNOWLEDGMENTS}

The author has profited from discussions with J. Cruz and J. Navarro-Salas. He acknowledges the Spanish MEC, CSIC, and IMAFF (Madrid) for a research contract. This work was partially supported by the Comisión Interministerial de Ciencia y Tecnología and DGICYT.
[1] A. Strominger, hep-th/9501071.

[2] R. B. Mann, Phys. Rev. D 47, 4438 (1993).

[3] T. Banks and M. O'Loughlin, Nucl. Phys. B362, 649 (1991).

[4] J. Gegenberg, G. Kunstatter, and D. Louis-Martines, Phys. Lett. B 321, 193 (1994); Phys. Rev. D 51, 1781 (1995); gr-qc/9501017.

[5] J. Cruz, J. Navarro-Salas, M. Navarro, and C. F. Talavera, Phys. Lett. B 402, 270 (1997); M. Navarro, Phys. Rev. D 56, 2384 (1997).

[6] J. Cruz, J. M. Izquierdo, D. J. Navarro, and J. Navarro-Salas,
Report No. FTUV-97-10, IFIC-97-10, hep-th/9704168.

[7] W. Kummer, gr-qc/9612016; W. Kummer and P. Widerin, Phys. Rev. D 52, 6965 (1995).

[8] P. Schaller and T. Strobl, Mod. Phys. Lett. A 9, 3129 (1994); in Low-Dimensional Models in Statistical Physics and Quantum Field Theory, Proceedings of the International Conference, Schladming, Austria, 1995, edited by H. Grosse and L. Pittner, Lecture Notes in Physics, Vol. 469 (Springer, Berlin, 1996), p. 321, hep-th/9507020. 